\title{
Rings containing a field of characteristic zero
}

\author{
DAVID JOHN BENSON
}

\begin{abstract}
Let $K$ be a field of characteristic zero, and let $R$ be a ring containing $K$. Then either $R^{\times}=K^{\times}$or $K^{\times}$is a subgroup of infinite index in $R^{\times}$.
\end{abstract}

This note was prompted by recent email exchanges with various colleagues. We use the term "ring" to mean a ring with identity, not necessarily commutative. For a ring $R$, denote by $R^{\times}$the multiplicative group of invertible elements of $R$.

Theorem. Let $K$ be a field of characteristic zero, and let $R$ be a ring containing $K$. Then either $R^{\times}=K^{\times}$or $K^{\times}$is a subgroup of infinite index in $R^{\times}$.

Proof. Suppose, to the contrary, that $\left|R^{\times}: K^{\times}\right|=m>1$. Letting $d=m$ !, there is a proper normal subgroup of index dividing $d$ in $R^{\times}$, and contained in $K^{\times}$. Choose $s \in R^{\times}, s \notin K^{\times}$. Then $s^{d}$ is some element of $K^{\times}$. Let $n \in \mathbb{Z} \subseteq K$ with $n^{d} \neq s^{d}$. Then

$$
(s-n)\left(s^{d-1}+n s^{d-2}+\cdots+n^{d-1}\right)=s^{d}-n^{d}
$$

is in $K^{\times}$, and hence $s-n$ has a multiplicative inverse. So $s-n \in R^{\times}$, and hence $(s-n)^{d} \in K^{\times}$.

Choose $d+1$ different values of $n$ with $n^{d} \neq s^{d}$, say $n_{0}, \ldots, n_{d}$. Then we have $\left(s-n_{i}\right)^{d} \in K^{\times}$for $i=0, \ldots, d$. Expanding out these equations, and using the fact that we are in characteristic zero, the non-vanishing of the Vandermonde determinant then implies that $1, s, \ldots, s^{d}$ are all in $K$. But $s$ was chosen not to be in $K$. This contradiction proves the theorem. 
Open Access. This article is distributed under the terms of the Creative Commons Attribution 4.0 International License (http://creativecommons.org/licenses/ by/4.0/), which permits unrestricted use, distribution, and reproduction in any medium, provided you give appropriate credit to the original author(s) and the source, provide a link to the Creative Commons license, and indicate if changes were made.

DAVID JoHn BENSON

Institute of Mathematics,

University of Aberdeen,

Fraser Noble Building,

Aberdeen AB24 3UE,

UK

e-mail: d.j.benson@abdn.ac.uk

Received: 25 August 2017 